\title{
The Study on Expression of Request Between Chinese and Japanese Based on Pragmatics
}

\section{Liying Mi}

School of Foreign Studies, Shanghai University of Finance and Economics, Shanghai, China

\section{Email address:}

mi.liying@mail.shufe.edu.cn

\section{To cite this article:}

Liying Mi. The Study on Expression of Request Between Chinese and Japanese Based on Pragmatics. Education Journal. Vol. 4, No. 5, 2015, pp. 298-302. doi: 10.11648/j.edu.20150405.27

\begin{abstract}
There is close relationship between pragmatics and the context which includes the most important scene elements. Scene element consists of the topic of the discourse, the effect on the counterpart, the age of the other side and the relationship between the two sides and so on. Request is a frequent speech act in daily life. So, what kind of differences can occur when requests between Chinese and Japanese are restricted by different contexts? This thesis makes statistical investigation and analysis onthe differences in the extent of politeness, expression, and utterance meaning between college students from China and Japan, from the perspective of context by employing the basic principles of pragmatics.
\end{abstract}

Keywords: Pragmatics, Expression of Request, the Extent of Politeness

\section{基于语用学的中日请求表达研究}

\section{米丽英}

外国语学院, 上海财经大学, 上海, 中国

\section{邮箱}

mi.1iying@mail.shufe.edu.cn

摘要: 语用学与语境有着密切的关系, 而语境中所包含的场景要素最为重要。场景要素包括话语现场的话题、对对方 产生的影响、对方的年龄以及和与对方的亲疏关系等等。请求是日常生活中频繁进行的语言行为。那么，中日两国人 的请求表达受语境的制约会存在何种差异? 本文运用语用学的基本原理, 从语境的角度分析并统计了中日大学生的请 求表达在礼貌程度、表现形式、话语意义方面的异同。

关键词: 语用学, 请求表达方式, 礼貌程度

\section{1. 引言}

选择合适的语言、讲究措辞礼貌是促进人际关系的必 要因素。在道兼欠、感谢、请求帮助的时候, 选择适当的措 辞, 便于建立和保持良好的人际关系。本文以中日大学生 请求帮助时所采用的表达方式作为研究对象, 分析了请求 表达方式的类型以及其中存在的礼貌程度的差异。

蒲谷 [1] 定义: 日语中将促使对方实施某种行为为目 的的表达方式统称为 “行为要求表现方式”。它包括 “命
令”、“请求”、“建议” 等, 现代汉语的祈使句中也有 相应句式。

说话人为了自己或自己一方向听话人请求某事时, 会 使用 “行为要求表现方式” 中的 “请求表达”。日语中将 这种请求表达方式称之为“依赖表现”。

一般来说, 请求方会使用委婉礼貌的语言来实现请求 目的。举个例子, 希望别人帮忙照张相片时, 不是使用「写 真撮ってくれ（给我照相）」，而是「すみません、写真 を撮りたいんですけどシャッタ一を押してもらっても いいですか? (不好意思, 我想照张相, 您能帮我按一下 
快门吗？）」; 「お忙しい所すみません。写真をとって いただけますか。（在您忙的时候不好意思打扰一下, 能 帮忙照一下相吗？）」、「悪いんだけど1枚写真とって もらえないかな？（不好意思，能帮忙照张相片吗？）」

中文中也有类似的表达方式, “我想照一张相, 你能 帮忙吗? ”、“不好意思, 能帮我按一下快门吗? ”等等。 由此可见, 中日请求表达的类型可谓丰富多彩, 如果通过 间接的表达方式, 以商量的口气提出请求的话, 令人易于 接受。或者借助辅助语来减缓话语的压力, 也能避免唐突。

山冈 [2]指出：“请求表达中如果措辞礼貌程度高的 话, 有利于与人保持良好的交际关系。涁涁有礼、友好和 谐的表达方式不会损坏人际关系。”

从20世纪70年代开始, 作为一种语用现象, 请求表达 中的措辞礼貌原则、表现形式、话语意义受到了关注。语 用学与语境、社会、文化、教育知识等因素有着密切的关 系, 而语境是影响请求表达方式的主要因素。本文运用语 用学的基本原理, 从语境的场景要素来分析请求表达方式 在实际生活中的运用情况。场景要素包括话语现场的话题、 对对方产生的影响、对方的年龄以及和与对方的亲疏关系 等等。

\section{2. 前期研究现状述评}

请求是日常生活中频繁进行的语言行为。中日两国人 的请求表达受语境的制约会存在何种差异? 礼貌程度有 何不同? 迄今为止, 对于请求表达的措辞礼貌程度或者表 现形式进行的研究如下:

费建华 [3] 论述了间接言语行为理论是实施间接请求 的理论依据, 分析了在日语中实施间接请求的语用策略。 桥本 [4]分析了“邀请人看演出时” 中日两国 “礼貌表现” 中礼貌程度的不同, 得到了日本人顾虑级别高低以及与对 方的亲疏关系, 使用的措辞礼貌程度差异明显, 而中国人 的措辞则个体差异很大这一结论。潘敏 [5]从语用学的角 度考察了日语请求表达方式中存在的具体语言结构以及 语言手段, 并运用言语行为等理论着重探讨了礼貌原则对 请求表达方式的影响。张红鹰 [6]通过调查电视剧中会话 场面的请求表达来详细考察日语口语中请求表达的使用 状况。总结归纳出了八种不同类型的请求表达形式。清水 [7]分析了男女不同性别的日本大学生使用请求表达时的 性别差异。结果表明: 男性对同性的学长礼貌程度较高, 对异性则礼貌程度更高。女性对朋友和学姐使用的请求表 达礼貌程度高。和男性对男性相比, 女性向异性寻求帮助 时, 所使用语言的礼貌程度相对较低, 而对同性的礼貌程 度较高。盧万才 $[8]$ 从二语习得及语言结构对比研究的角 度, 结合中日两国的文化背景、语言构造的差异, 考察了 日本人和日语学习者在请求帮助时使用的表达形式的特 点及礼貌程度的深浅, 分析了不同于日语所特有的语言行 为及使用规则。于沝 [9]将间接请求表达继续分为规约性 间接请求与非规约性间接请求, 着重运用语用学中礼貌原 则理论探讨其对请求表达方式的影响, 并从文化角度分析 了请求表达存在差异的原因。

以上研究都取得了优秀的研究成果, 学者们对请求表 达这种语言现象描述得比较详尽, 让学习者和读者加深了
对它的理解。本文在对前期研究归纳总结的基础上, 运用 语用学的基本原理, 从语境的角度来分析并统计了中日大 学生的请求表达在礼貌程度、表现形式、话语意义方面的 异同。

\section{3. 调查概要}

\section{1. 调查对象}

调查对象为中日在校硕士研究生和大学生。调查问卷 分为中文版和日文版两种。调查内容设定了请求、感谢、 提醒、道兼等几个场面, 被调查者用本国母语来自由回答 书面提问。调查问卷上还有除请求表达之外的提醒表达、 感谢表达等等项目, 但本文只将请求表达作为研究对象进 行分析。有效问卷日本为705份、中国为 272 份。

回答问卷调查者的数据如下所示。其中日本人以J、 中国人以C、男性以M、女性以F来表示。日本男性以JM、 中国男性CM、日本女性JF、中国女性以CF来表示。

表1 有效调查问卷数。

\begin{tabular}{lll}
\hline & 日本 $(\mathrm{J})$ & 中国 $(\mathrm{C})$ \\
\hline 男性 $(\mathrm{M})$ & 202 & 169 \\
女性 $(\mathrm{F})$ & 503 & 103 \\
总数 & 705 & 272 \\
\hline
\end{tabular}

\section{2. 调查项目}

调查问卷中请求表达的提问内容如下:

表2 请求表现的提问内容。

[场面设定 ]

您和朋友一起去许久未去的自家附近的名胜古迹。两个人决定一起照 张相, 必须拜托谁来按一下快门。此时, 附近正好有三位初中生。假 设那时您想拜托其中的一个男孩来帮您照相。

（1）这时, 您会怎样拜托这位初中男生帮忙拍照呢? 请将您使用的语 言写下来。

结果, 那位男生发现您想要人帮忙拍照, 主动帮您拍了照。然后, 带 着微笑把照相机还给了您。那时, 要是您想向那位初中男生说点什么, 您会说些什么?

（2）这时, 正好附近还有一对中年夫妇。那时, 您想拜托那位男性为 您拍照, 您会怎样拜托? 请将您所使用的语言写下来。

\section{4. 分析方法}

请求某人做某事时采用的请求表达方式各式各样, 本 文在参考柳慧政 [10]对请求表达分类的基础之上, 重新将 此进行了分类。首先分为直接型、间接型两大类。直接型 是带有命令或指示语气的「して」「してください」型。 间接型是「やりもらい」的授受表达方式, 有「してくれ る」「してもらう」「していただく」等形式。

间接型下面再细分为授受型、可能型、许可型、愿望 型、回避型6种类型。其中可能型有「いける? 」「でき る?」「おねがいできる?」等几种形式。

许可型是以商量的口气提出请求, 希望得到听话人的 许可, 有「していただいてもよろしい」「してもらって もいい」等形式。 
愿望型是希望对方为自己采取某项行动, 有「してほ しい」「してほしいんだけど」「したいんだけど」等表 达方式。清水 [7]中指出, 回避型和说明型与愿望型一样, 因为请求方的表达比较婉转含蓄, 被请求方有必要推测请 求方所请求的内容。具体比较一下它们之间的差异, 可知 回避型不使用直接的请求方式, 用暗示法来代替。通过暗 示语句来表达相关意思, 以免直接驱使对方, 给对方造成 压力, 而使对方感到为难。请求者对自身的行动和状况加 以说明, 希望对方达到满足自己愿望和要求的则属于说明 型。

通常情况下，中文的请求表达可分为执行句、疑问句、 愿望句、命令句、条件句五类，根据该分类与此次调查得 到的数据, 本文将中文的请求表达予以重新分类。

具体分类形式如下表所示:

表3 中文请求表达的重新分类。

\begin{tabular}{|c|c|c|}
\hline 分类 & 中文 & 日文 \\
\hline \multirow{4}{*}{$\begin{array}{l}\text { 执行句 } \\
\text { (直接型) }\end{array}$} & 请V & てください \\
\hline & 拜托V & お願いします \\
\hline & 帮我V & 手伝ってください \\
\hline & 能……吗？ & てくれますか \\
\hline \multirow{4}{*}{$\begin{array}{l}\text { 疑问句 } \\
\text { (授受型) }\end{array}$} & 能麻烦……吗？ & ていただけますか \\
\hline & 不能 $V$ 我……吗？ & てもらえますか \\
\hline & 可以V我……吗? & てくださいますか \\
\hline & 我想请 & ていただきたい \\
\hline 愿望句 & 我想 & てもらいたい \\
\hline \multirow{2}{*}{ (愿望型) } & 希望V & お願いしたい \\
\hline & 需要V & てほしい \\
\hline 征求文 & 不知你愿不愿意 $\mathrm{V} ?$ & お願いしてもょろしい？ \\
\hline （许可型） & ……v好吗？ & してもらってもいいですか \\
\hline 可能句 & 能不能麻烦 $\mathrm{V} \cdot \cdots \cdot .$. & お願いできるでしょうか \\
\hline (可能型) & 可不可以V…... & お願いできますか。 \\
\hline
\end{tabular}

\section{5. 调查分析结果}

日本大学生和中国大学生面向中学生和中年男性拜 托照相时, 究竟用了这样的请求表达? 本文对此进行了 调查。根据调查数据对它进行分类统计, 得到了以下结 论:

\section{1. 日本大学生的数据分析结果}

针对日本大学生展开的调查数据分析结果如表 4 和表 5所示:

表4 日本大学生直接型的使用率。

\begin{tabular}{ll}
\hline 请求对象 & 直接性 \\
\hline 中学生 & 14 \\
& $2.0 \%$ \\
中年男性 & 16 \\
\hline
\end{tabular}

表5 日本大学生间接型的使用率。

\begin{tabular}{lllll}
\hline 请求对象 & 授受型 & 许可型 & 愿望型 & 可能型 \\
\hline \multirow{2}{*}{ 中学生 } & 260 & 235 & 20 & 13 \\
& $36.9 \%$ & $33.3 \%$ & $2.8 \%$ & $1.8 \%$ \\
中年男性 & 293 & 250 & 25 & 23 \\
& $41.6 \%$ & $35.5 \%$ & $3.5 \%$ & $3.3 \%$ \\
\hline
\end{tabular}

分析日本的调查结果，除回避型和说明型无一例出现 之外, 其他形式均有使用, 请求表现的形式丰富多样。由 表4可见, 日本大学生面向中学生和中年男性, 使用直接 型的比例很低, 分别只占 $2.0 \%$ 和 $2.3 \%$ 。而间接型的请求表 达方式使用的频率很高, 其中授受型和许可型使用最多, 其次为愿望型和可能型。这几类请求方式均从属于间接型。 具体使用率见表 5 。

更细致观察的话, 日本大学生向中学生请求帮助时, 和隶属于间接型的可能型相比, 更倾向于使用直接型。但 是, 向中年男性请求帮助时, 和直接型相比, 更倾向于使 用可能型。

于是得出了一个结论, 当对方年长时, 日本大学生倾 向使用对方方便拒绝、或者给对方拒绝留有余地、减轻对 方的心理压力的间接型, 而直接了断、礼貌级别较低、可 能会冒犯对方的直接型不太被采用。

从整体来看, 请求对象无论是比自己辈分低的中学生 还是比自己辈分高的中年男性, 日本大学生都不倾向使用 直接型的请求表达方式。可见日本大学生为了维护良好的 交际关系, 为了避免给对方强加与人的感觉, 充分给予对 方选择帮与不帮的自由, 并通过语言充分表达自己对对方 尊敬的感情。可见, 只有使用了让被请求方容易接受的请 求表达方式, 自己的请求行为才容易实现。

\section{2. 中国大学生的数据分析结果}

针对日本大学生展开的调查数据分析结果如表 6 和表 7所示:

表6 中国大学生直接型的使用率。

\begin{tabular}{ll}
\hline 请求对象 & 直接性 \\
\hline 中学生 & 9 \\
& $3.3 \%$ \\
中年男性 & 11 \\
\hline
\end{tabular}

表7 中国大学生间接型的使用率。

\begin{tabular}{lllll}
\hline 请求对象 & 授受型 & 许可型 & 愿望型 & 可能型 \\
\hline \multirow{2}{*}{ 中学生 } & 141 & 46 & 4 & 11 \\
& $51.8 \%$ & $16.9 \%$ & $1.5 \%$ & $4.0 \%$ \\
中年男性 & 106 & 31 & 2 & 6 \\
& $39.0 \%$ & $11.4 \%$ & $0.7 \%$ & $2.2 \%$ \\
\hline
\end{tabular}

中国大学生的请求表达中, 回避型和说明型也无一例 出现, 而其他形式均有使用。这点和日本的调查结果一样。

由表 6 可知, 中国大学生面向中学生和中年男性时直 接型的使用率仅为 $3.3 \%$ 和 $4.0 \%$ 。可知中国大学生均喜欢 使用礼貌程度高的间接型请求表达方式。其中授受型和许 可型使用最多。但是和日本大学生不同的是, 愿望型的使 用高于可能型。这几类请求方式均从属于间接型。

当被请求对象为初中学生时, 中国大学生更倾向于使 用直接型, 而不喜欢使用可能型。与此相反, 向中年男性 请求帮助时, 和直接型相比, 更倾向于使用可能型。这点 也与日本大学生存在较大的差异。具体情况可见表7。

由此可见, 中国大学生为了缩小相互的距离, 为了向 辈分小的人表示亲切信赖的感情, 和疏远有距离的间接型 
相比, 更喜欢采用给人以亲切感的直接型。但是, 当被请 求对象为长辈时, 为了表示尊敬的程度而使用间接型。这 一点通过具体数据得到了证实。

\section{3. 中日大学生调查数据的比较}

通过对中日两国大学生调查数据的比较, 得到的结果 如表8和表9所示:
表8 中日大学生直接型的使用率。

\begin{tabular}{lll}
\hline 调查对象 & 直接型(日本) & 直接型 (中国) \\
\hline 中学生 & $2.0 \%$ & $3.3 \%$ \\
中年男性 & $2.3 \%$ & $4.0 \%$ \\
\hline
\end{tabular}

表9 中日大学生间接型的使用率。

\begin{tabular}{|c|c|c|c|c|c|c|c|c|}
\hline 请求对象 & 授受型 (日本) & 授受型 (中国) & 许可型 (日本) & 许可型 (中国) & 愿望型 (日本) & 愿望型 (中国) & 可能型 (日本) & 可能型 (中国) \\
\hline 中学生 & $36.9 \%$ & $51.8 \%$ & $33.3 \%$ & $16.9 \%$ & $2.8 \%$ & $1.5 \%$ & $1.8 \%$ & $4.0 \%$ \\
\hline 中年男性 & $41.6 \%$ & $39.0 \%$ & $35.5 \%$ & $11.4 \%$ & $3.5 \%$ & $0.4 \%$ & 3. $3 \%$ & $2.2 \%$ \\
\hline
\end{tabular}

表 8 和表 9 为各种类型的请求表达方式的使用频率表。

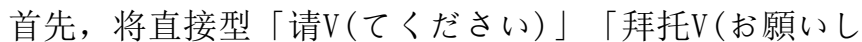
ます)」「帮我 $V$ (手伝ってください)」的使用频率相比较 可知, 日本大学生的请求对象为中学生和中年男性时, 分 别为 $2.0 \%$ 和 $2.3 \%$ 。而中国大学生分别为 $3.3 \%$ 和 $4.0 \%$, 比 日方的比率稍高一些。

为什么能得到这样的结果? 语言是文化的载体, 这应 该和中日文化的不同有着重要的关系。日本人无论对方辈 分高低, 只要是初次见面, 就会保持适当的距离, 之后再 逐渐加深友谊。但是中国人刚好和日本人相反，从一开始 就向对方表示亲切感, 希望尽快建立相互之间的信赖关系。

总体而言, 无论是中国还是日本大学生, 主要倾向于 使用授受型和许可型两种请求表达方式。许可型中的 “不 知你愿不愿意 $\mathrm{V}$ ? ”、“..... V 好吗? ”相比, 中国大学生 更倾向于使用授受型的“能……吗？”、“能麻烦……吗？”、 “不能 $V$ 我......吗？”、“可以 $V$ 我……吗？”

汉语中的授受型给人一种柔和亲切的感觉, 比许可型 更方便使用。而日本大学生为什么会倾向于使用许可型呢? 从场景要素的人际关系、心理关系的角度分析可知: 日本 大学生对辈分的意识不是很强, 既要表示对被请求方的尊 敬, 还不能过于亲近, 需要保持恰到好处的距离感的情况 之下, 许可型的表达方式更为合适。这说明与辈分关系相 比，他们更注重人际交往中的亲疏关系。

此外, 预想不到的是: 和中国大学生相比, 日本大学

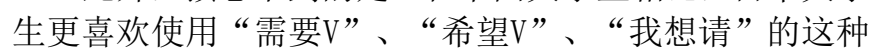
愿望型的请求表达方式, 所占比率高达 $2.8 \%$ 和 $3.5 \%$ 。而中 国大学生当被请求对象为初中生时, 愿望型的使用比率为 $1.5 \%$, 中年男性时, 所占比率仅为 $0.4 \%$ 。可见中国大学生 对年长者尽量避免使用愿望型, 可能因为愿望型直接了断 地说出了自己的愿望, 相比之下礼貌级别不高, 所以对年 长者采取了慎用的态度。

\section{6. 结束语}

从语用学的角度来看, 场景包括话语现场的话题、 对对方产生的影响、对方的年龄以及和与对方的亲疏关 系等等。在限定的场景之下, 为了让对方能够接受自己 的请求, 需要运用怎样的策略才能够达到请求的目的? 因此请求时的表现手段和礼貌程度显得尤为重要, 成为 了使交际活动顺利进行的重要因素。因此, 本文重点分
析了中日大学生请求表达中的表现手段和礼貌程度, 结 论如下:

1) 整体来看, 无论是日本还是中国大学生, 向晚辈或长 辈请求帮助时, 大部分人采用授受型与许可型两种方 式。这两种表达方式语气委婉让人容易接受, 都隶属 于 “间接型”。

2) 再看 “直接型” 的使用情况。采用 “直接型” 的人数 显然比 “间接型” 少得多。总体而言, 和日本大学生 相比, 中国大学生面向比自己年龄小的中学生提出请 求时，有一部分人倾向使用 “亲切称呼十直接型” 的表达方式。请求的时候加入对对方的亲切称呼, 拉 近距离, 推动对方接受请求。

3）而当对方为长辈时, 中国大学生则更喜欢使用礼貌程 度高的 “间接型” 请求表达, 这说明中国大学生对长 辈措辞慎重、比较重视辈分关系。

4) 日本大学生则无论对方辈分高低, 都会采用礼貌程度 高、让对方容易推辞的表达方式。这说明与辈分关系 相比，他们更注重人际交往中的亲疏关系。

请求表达现象普遍存在于各种不同的文化和语言之 中, 制约并协调着人们的交际活动。请求者需要顾虑当时 的场景要素来采取相应的请求语言行为, 这在交际活动中 已经成为一项语言规律。

\section{致谢}

本文为教育部留学回国人员科研启动基金课题 (项目 编号：2010120010）《基于super-function 的中日被动 句机器翻译研究》的阶段性成果之一。

本文为青年教师预研究项目一基本科研业务费 (项目 编号: 2013110037) 《基于文本挖掘技术的中日礼貌措辞 研究》的阶段性成果之一。

\section{参考文献}

［1］蒲谷宏・川口義一 ・坂本恵.『敬語表現』[M]. 大修館書店, 1998:1-101。

[2] 山岡政紀・『発話機能論』[M].くろしお出版, 2008:1-98。 
[3] 费建华. 日语间接请求及其语用策略. 解放军外国语学院学 报 $[J] ， 2002$, vol.25(1):42-46。

［4］橋本永貢子 ·三井栄.「日中の待遇表現に関わる”ものさ し"について - アンケート調査の統計的分析から-」岐阜 大学地域科学部研究報告21 [R]. 岐阜大学, 2007:99-110。

[5] 潘敏. 对日语中的请求表达的语用考察. 广西师范大学硕士 学位论文 [D], 2008: 1-54。

[6] 张红鹰. 关于请求表达使用状况的考察——以电视剧为中 心. 大连海事大学硕士学位论文 [D], 2009:1-61。
［7］清水勇吉.「依頼表現に見るポライトネス:性差のかかわ りを中心に」. 徳島大学国語国文學 [J]. Vol.22, 2009:53-35。

［8］盧万才.「依頼表現形式の丁寧度に関する日中比較一中国 人日本語学習者習得状況調査一」. 中日言語対照研究論叢 第2号 [Z]. 北京大学出版社, 2011:307-317。

[9] 于永. 对日语请求表达方式的语用研究. 辽宁大学硕士学 位学位 [D], 2013:1-44。

[10］柳慧政.「依頼談話の日韓対照研究: 談話の構造・ストラ テジーの観点から」。『笠間書院』[R]，2012:1-150。 